\title{
EFFICIENT ESTIMATOR FOR POPULATION MEAN IN STRATIFIED DOUBLE SAMPLING IN THE PRESENCE OF NONRESPONSE USING ONE AUXILIARY VARIABLE
}

\author{
A.E. Anieting1, E. I. Enang ${ }^{2}$ and C. E. Onwukwe ${ }^{2}$ \\ ${ }^{1}$ Department of Statistics, University of Uyo, Uyo, Nigeria \\ ${ }^{2}$ Department of Statistics, University of Calabar, Nigeria \\ Email: akaninyeneanieting@uniuyo.edu.ng.
}

\section{Cite this article:}

A.E. Anieting, E. I. Enang, C. E. Onwukwe (2021), Efficient Estimator for Population Mean in Stratified Double Sampling in the Presence of Nonresponse Using One Auxiliary Variable. African Journal of Mathematics and Statistics Studies 4(2), 40-50. DOI: 10.52589/AJMSSYF4V11QV.

\section{Manuscript History}

Received: 28 April 2021

Accepted: 18 May 2021

Published: 22 May 2021

Copyright $(9) 2020$ The Author(s). This is an Open Access article distributed under the terms of Creative Commons AttributionNonCommercial-NoDerivatives 4.0 International (CC BY-NC-ND 4.0 ), which permits anyone to share, use, reproduce and redistribute in any medium, provided the original author and source are credited.
ABSTRACT: A modified form of the population mean estimator suggested by Anieting and Enang (2020) in stratified double sampling in the presence of nonresponse using a single auxiliary variable has been proposed. The Mean Squared Error (MSE) and the bias of the proposed estimator have been given using large sample approximation. The empirical study shows that the MSE of the suggested estimator is more efficient than all other existing estimators in the same scheme. Determination of the optimal values of the first and second phases samples has also been done

KEYWORDS: Efficiency, Nonresponse, Stratified Double Sampling

MSC: 62D05. 


\section{INTRODUCTION}

Classical double sampling for stratification is a method that uses two random samples where the second sample is a stratified subsample of the first sample. If the problem of nonresponse is there then this subsample may be divided into classes of respondents and non-respondents (Cochran (1977)). It is resorted to when the auxiliary variable $\mathrm{x}$ needed for stratification of the population units is not available prior to sampling and the frequency distribution of $\mathrm{x}$ and the stratum weights are not known in advance. The presence of nonresponse distorts parameter estimation by increasing bias in estimates resulting in larger variances. In a welldesigned research, handling nonresponse is important and several authors have tried to tackle this problem differently. According to Singer (2006) statisticians have been concerned mainly with imputation and weighting as ways of adjusting for the bias caused by nonresponse while social scientists have tended to focus on measuring, understanding and reducing the nonresponse rates themselves.

Hansen and Hurwitz (1946) suggested a method of sub-sampling of non-respondents so as to modify the non-response in their mail surveys. Khoshnevisan et al. (2007), Chaudhary et al. (2009), Chaudhary and Singh (2013), Chaudhary and Kumar (2015) and Anieting and Enang 2020) have all suggested different estimators in two-phase stratified sampling under nonresponse. In this article, a modified efficient ratio-product estimator in stratified double sampling under nonresponse using one auxiliary variable is been proposed. The characteristics of the estimator suggested have been given.

\section{Sampling Design}

Consider a population of size $\mathrm{N}$ divided into k strata. Let the size of the $i^{\text {th }}$ stratum be $N_{i}$ ( $\mathrm{i}=1,2, \ldots . \mathrm{k})$ such that $\sum_{i}^{k} N_{i}=\mathrm{N}$. A large first sample of size $n_{i}^{\prime}$ is drawn from $N_{i}$ units by (SRSWOR) scheme for the $i^{\text {th }}$ stratum and auxiliary variable $\bar{x}_{i}^{\prime}$ is observed to estimate the population mean $\bar{X}$, which is unknown. Secondly, a smaller second phase sample of size $n_{i}$ is drawn from $n_{i}^{\prime}$ unit by SRSWOR. Let $\mathrm{Y}$ be the study variable with population mean $\bar{Y}$ $=\sum_{i}^{k} p_{i} \bar{Y}_{i}\left(\bar{Y}_{i}\right.$ being the mean of the $i^{t h}$ stratum based on $N_{i}$ units and $\left.p_{i}=\frac{N_{i}}{N}\right)$ and assume that the non-response is observed on the study variable at second phase while the auxiliary variable is free from non-response. Also, assume that there are $n_{i 1}$ respondent units and $n_{i 2}$ non-respondent units in $n_{i}$ units at the second phase. Applying Hansen and Hurwitz (1946) technique of sub-sampling of non-respondents, a sub-sample of $h_{i 2}=\frac{n_{i 2}}{L_{i}}, L_{i} \geq 1$ unit is selected from the sample of $n_{i 2}$ non-respondents and the information is collected from all of them.

\section{Some Existing Estimators}

Some existing estimators for population mean in stratified double sampling with single auxiliary variable in the presence of non-response shall be presented.

\section{Khare and Srivastava (1997) Estimator}

Khare and Srivastava (1997) proposed two ratio estimators given as

$T_{1}^{*}=\bar{y}_{s t}^{*} \frac{\bar{x}_{s t}^{\prime}}{\bar{x}_{s t}}$ 
with mean square error,

$\operatorname{MSE}\left(T_{1}^{*}\right)=\sum_{i}^{k} f_{i}^{\prime} p_{i}^{2} S_{Y_{i}}^{2}+\sum_{i}^{k} f_{i}^{*} p_{i}^{2}\left(S_{Y_{i}}^{2}+R^{2} S_{X_{i}}^{2}-2 R \rho_{i} S_{X_{i}} S_{Y_{i}}\right)+\sum_{i}^{k} \frac{\left(L_{i}-1\right)}{n_{i}} W_{i 2} P_{i}^{2} S_{Y_{i 2}}^{2}$

Where $f_{i}^{\prime}=\left(\frac{1}{n_{i}^{\prime}}-\frac{1}{N_{i}}\right), f_{i}^{*}=\left(\frac{1}{n_{i}}-\frac{1}{n_{i}^{\prime}}\right)$ and

$T_{2}^{*}=\bar{y}_{s t}^{*} \frac{\bar{x}_{s t}}{\bar{x}_{s t}^{\prime}}$

With mean square error,

$\operatorname{MSE}\left(T_{1}^{*}\right)=\sum_{i}^{k} f_{i}^{\prime} p_{i}^{2} S_{Y_{i}}^{2}+\sum_{i}^{k} f_{i}^{*} p_{i}^{2}\left(S_{Y_{i}}^{2}+R^{2} S_{X_{i}}^{2}-2 R \rho_{i} S_{X_{i}} S_{Y_{i}}\right)+\sum_{i}^{k} \frac{\left(L_{i}-1\right)}{n_{i}} W_{i 2} P_{i}^{2} S_{Y_{i 2}}^{2}$

Where $f_{i}^{\prime}=\left(\frac{1}{n_{i}^{\prime}}-\frac{1}{N_{i}}\right), f_{i}^{*}=\left(\frac{1}{n_{i}}-\frac{1}{n_{i}^{\prime}}\right)$

\section{Chaudhary et al. (2009) Estimator}

Chandhary et. al. (2009) suggested a family of combined-type estimators given as

$T_{C}=\bar{y}_{s t}^{*}\left[\frac{a \bar{X}+b}{\alpha\left(a \bar{X}_{s t}+b\right)+(1-\alpha)(a \bar{X}+b)}\right]^{g}$

Where $\bar{X}_{s t}=\sum_{i}^{k} p_{i} \bar{x}_{i}$ and $\bar{X}=\sum_{i}^{k} p_{i} \bar{X}_{i} ; \bar{x}_{i}$ and $\bar{X}_{i}$ are respectively mean based on $n_{i}$ units and mean based on $N_{i}$ in the $i^{\text {th }}$ stratum for auxiliary variable

They gave mean square error as

$\operatorname{MSE}\left(T_{C}\right)=\sum_{i}^{k} f_{i} p_{i}^{2}\left[S_{Y_{i}}^{2}+\alpha^{2} \lambda^{2} g^{2} R^{2} S_{X_{i}}^{2}-2 \alpha \lambda g R \rho_{i} S_{X_{i}} S_{Y_{i}}\right]+\sum_{i}^{k} \frac{\left(L_{i}-1\right) W_{i 2} P_{i}^{2} S_{Y_{i 2}}^{2}}{n_{i}}$

Where $f_{i}=\left(\frac{1}{n_{i}}-\frac{1}{N_{i}}\right), \lambda=\frac{a \bar{X}}{a \bar{X}+b}$,

\section{Chaudhary et al. (2012) Estimator}

Chaudhary et al. (2012) proposed a combined type estimator given as

$T_{F c}(\alpha)=\bar{y}_{s t}^{*}\left[\frac{(A+C) \bar{X}+f B \bar{X}_{s t}}{(A+B) \bar{X}+C \bar{X}_{s t}}\right]$

Where $\mathrm{A}=(\alpha-1)(\alpha-2), \mathrm{B}=(\alpha-1)(\alpha-4), \mathrm{C}=(\alpha-3)(\alpha-2)(\alpha-4), \alpha>0, \mathrm{f}=\frac{n_{i}^{\prime}}{N_{i}}$

The mean square error as obtained by them is

$\operatorname{MSE}\left(T_{F c}(\alpha)\right)=\sum_{i}^{k}\left(\frac{1}{n_{i}}-\frac{1}{N_{i}}\right) P_{i}^{2}\left[S_{Y_{i}}^{2}+\emptyset(\alpha)^{2} R S_{X_{i}}^{2}-2 \emptyset(\alpha) R \rho_{i} S_{X_{i}} S_{Y_{i}}\right]$ 
Where $\varnothing(\alpha)=\frac{C-f B}{A+f B+C}$

\section{Chandhary And Kumar (2015) Estimator}

A combined type estimator was proposed by Chandhary and Kumar (2015) as follows:

$$
T_{c}^{\prime}=\bar{y}_{s t}^{*}\left[\frac{a \bar{x}_{s t}^{\prime}+b}{\alpha\left(a \bar{x}_{s t}+b\right)+(1-\alpha)\left(a \bar{x}_{s t}^{\prime}+b\right)}\right]^{g}
$$

With mean square error given as

$\operatorname{MSE}\left(T_{c}^{\prime}\right)=\sum_{i}^{k} f_{i}^{\prime} p_{i}^{2} S_{y_{i}}^{2}+\sum_{i}^{k} f_{i}^{*} p_{i}^{2}\left(S_{y_{i}}^{2} \quad+g^{2} \lambda^{2} R^{2} \alpha^{2} S_{x_{i}}^{2}-2 g \lambda R \alpha p_{i} S_{x_{i}} S_{y_{i}}\right)+$ $\sum_{i}^{k}\left(\frac{L_{i}-1}{n_{i}}\right) W_{i 2} p_{i}^{2} S_{y_{i}}^{2}$

\section{Anieting and Enang (2020) Estimator}

A product-ratio estimator was suggested by Anieting and Enang (2020) as follows

$T_{a e}=\bar{y}_{s t}^{*}\left(\frac{\bar{x}_{s t}^{\prime}+\varphi}{\bar{x}_{s t}-\varphi}\right)$

Where $\varphi=\sum_{i}^{k} C_{x_{i}}$ where $C_{x_{i}}$ is the coefficient of variation of the auxiliary variable. $\bar{y}_{s t}^{*}=$ $\sum_{i}^{k} p_{i} \bar{y}_{i}^{*} ; \bar{y}_{i}^{*}=\frac{n_{i 1} \bar{y}_{n_{i 1}}+n_{i 2} \bar{y}_{h_{i 2}}}{n_{i}}, p_{i}=\frac{N_{i}}{N}$

$\bar{y}_{n_{i 1}}$ and $\bar{y}_{h_{i 2}}$ are the means based on $n_{i 1}$ respondent units and $h_{i 2}$ subsampled nonrespondent units respectively for the study variable and $\bar{x}_{s t}^{\prime}=\sum_{i}^{k} p_{i} \bar{x}_{i}^{\prime}, \bar{x}_{s t}=\sum_{i}^{k} p_{i} \bar{x}_{i}$

with mean squared error given as

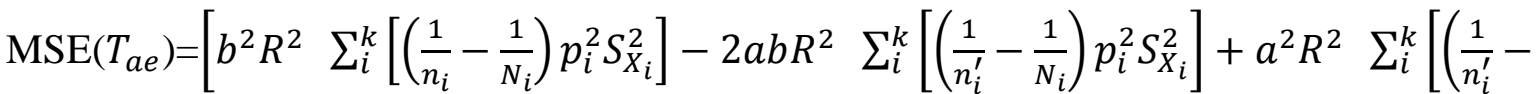

$\left.\left.\frac{1}{N_{i}}\right) p_{i}^{2} S_{X_{i}}^{2}\right]-2 b R \sum_{i}^{k}\left(\frac{1}{n_{i}}-\frac{1}{N_{i}}\right) p_{i}^{2} \rho_{i} S_{X_{i}} S_{Y_{i}}+2 a R \sum_{i}^{k}\left(\frac{1}{n_{i}^{\prime}}-\frac{1}{N_{i}}\right) p_{i}^{2} \rho_{i} S_{X_{i}} S_{Y_{i}}+\sum_{i}^{k}\left[\left(\frac{1}{n_{i}}-\right.\right.$

$\left.\left.\left.\frac{1}{N_{i}}\right) p_{i}^{2} S_{Y_{i}}^{2}+\frac{\left(L_{i}-1\right)}{n_{i}} p_{i}^{2} W_{i 2} S_{Y_{i 2}}^{2}\right]\right]$

Where $\mathrm{R}=\frac{\bar{Y}}{\bar{X}}, \mathrm{a}=\frac{\bar{X}}{\bar{X}+\varphi}$ and $\mathrm{b}=\frac{\bar{X}}{\bar{X}-\varphi}$

\section{Proposed Estimator and its properties}

The proposed estimator for population mean in stratified double sampling in the presence of nonresponse using single auxiliary variable is given as,

$T_{a e 1}=\bar{y}_{s t}^{*}\left(\frac{\bar{x}_{s t}^{\prime}-\rho_{i}}{\bar{x}_{s t}-\rho_{i}}\right)$ 
where $\rho_{i}$ is the correlation coefficient between $\mathrm{y}$ and $\mathrm{x}$.

$$
\bar{y}_{s t}^{*}=\sum_{i}^{k} p_{i} \bar{y}_{i}^{*} ; \bar{y}_{i}^{*}=\frac{n_{i 1} \bar{y}_{n_{i 1}}+n_{i 2} \bar{y}_{h_{i 2}}}{n_{i}}, p_{i}=\frac{N_{i}}{N}
$$

$\bar{y}_{n_{i 1}}$ and $\bar{y}_{h_{i 2}}$ are the means based on $n_{i 1}$ respondent units and $h_{i 2}$ subsampled nonrespondent units respectively for the study variable and $\bar{x}_{s t}^{\prime}=\sum_{i}^{k} p_{i} \bar{x}_{i}^{\prime}, \bar{x}_{s t}=\sum_{i}^{k} p_{i} \bar{x}_{i}$

\section{The Bias and the Mean Squared Error MSE) of the proposed Estimator.}

\section{The Bias of $T_{a e 1}$}

To obtain the bias of the proposed estimator, the following terms are defined:

$\bar{y}_{s t}^{*}=\bar{Y}\left(1+e_{0}\right), \bar{x}_{s t}=\bar{X}\left(1+e_{1}\right), \bar{x}_{s t}^{\prime}=\bar{X}\left(1+e_{2}\right)$ where the $e_{i}$ s are the relative error terms defined as

$e_{0}=\frac{\bar{y}_{s t}^{*}-\bar{Y}}{\bar{Y}}, e_{1}=\frac{\bar{x}_{s t}-\bar{X}}{\bar{X}}, e_{2}=\frac{\bar{x}_{s t}^{\prime}-\bar{X}}{\bar{X}}$

(1) becomes

$T_{a e 1}=\bar{Y}\left(1+e_{0}\right)\left(1+b e_{2}\right) \quad\left(1+b e_{1}\right)^{-1}$ where $\mathrm{b}=\frac{\bar{X}}{\bar{X}-\rho_{i}}$

$=\bar{Y}\left(1+e_{0}\right)\left(1+b e_{2}\right) \quad\left(1-b e_{1}+b^{2} e_{1}^{2}\right)$

$=\bar{Y}\left(1+e_{0}\right)\left[1-b e_{1}+b^{2} e_{1}^{2}+b e_{2}-b^{2} e_{1} e_{2}\right]$

$=\bar{Y}\left[1-b e_{1}+b^{2} e_{1}^{2}+b e_{2}-b^{2} e_{1} e_{2}+e_{0}-b e_{0} e_{1}+b e_{0} e_{2}\right]$ where the $e_{i} \mathrm{~s}$ are neglected having power more than two. Thus

$\operatorname{Bias}\left(T_{a e 1}\right)=\mathrm{E}\left(T_{a e 1}-\bar{Y}\right)$

$T_{a e 1}-\bar{Y}=\bar{Y}\left[-b e_{1}+b^{2} e_{1}^{2}+b e_{2}-b^{2} e_{1} e_{2}+e_{0}-b e_{0} e_{1}+b e_{0} e_{2}\right]$

$\operatorname{Bias}\left(T_{a e 1}\right)=\bar{Y}\left[b^{2} E\left(e_{1}^{2}\right)-b^{2} E\left(e_{1} e_{2}\right)-b E\left(e_{0} e_{1}\right)+b E\left(e_{0} e_{2}\right)\right]$

Since $\mathrm{E}\left(e_{0}\right)=\left(e_{1}\right)=\left(e_{2}\right)=0$

By substituting for the following expectations

$\mathrm{E}\left(e_{0}\right)=\left(e_{1}\right)=\left(e_{2}\right)=0$

$\mathrm{E}\left(e_{0}^{2}\right)=\frac{1}{\bar{Y}^{2}} \sum_{i}^{k}\left[\left(\frac{1}{n_{i}}-\frac{1}{N_{i}}\right) p_{i}^{2} S_{Y_{i}}^{2}+\frac{\left(L_{i}-1\right)}{n_{i}} p_{i}^{2} W_{i 2} S_{Y_{i 2}}^{2}\right]$

$\mathrm{E}\left(e_{1}^{2}\right)=\frac{1}{\bar{X}^{2}} \sum_{i}^{k}\left[\left(\frac{1}{n_{i}}-\frac{1}{N_{i}}\right) p_{i}^{2} S_{X_{i}}^{2}\right]$

$\mathrm{E}\left(e_{2}^{2}\right)=\frac{1}{\bar{X}^{2}} \sum_{i}^{k}\left[\left(\frac{1}{n_{i}^{\prime}}-\frac{1}{N_{i}}\right) p_{i}^{2} S_{X_{i}}^{2}\right]=\mathrm{E}\left(e_{1} e_{2}\right)$

$\mathrm{E}\left(e_{0} e_{1}\right)=\frac{1}{\bar{X} \bar{Y}} \sum_{i}^{k}\left(\frac{1}{n_{i}}-\frac{1}{N_{i}}\right) p_{i}^{2} \rho_{i} S_{X_{i}} S_{Y_{i}}$ 
$\mathrm{E}\left(e_{0} e_{2}\right)=\frac{1}{\bar{X} \bar{Y}} \sum_{i}^{k}\left(\frac{1}{n_{i}^{\prime}}-\frac{1}{N_{i}}\right) p_{i}^{2} \rho_{i} S_{X_{i}} S_{Y_{i}}$

$\operatorname{Bias}\left(T_{a e 1}\right)=\bar{Y}\left[\left(\frac{\bar{X}}{\bar{X}-\rho_{i}}\right)^{2} \frac{1}{\bar{X}^{2}} \sum_{i}^{k}\left[\left(\frac{1}{n_{i}}-\frac{1}{N_{i}}\right) p_{i}^{2} S_{X_{i}}^{2}\right]-\left(\frac{\bar{X}}{\bar{X}-\rho_{i}}\right)^{2} \frac{1}{\bar{X}^{2}} \sum_{i}^{k}\left[\left(\frac{1}{n_{i}^{\prime}}-\frac{1}{N_{i}}\right) p_{i}^{2} S_{X_{i}}^{2}\right]-\right.$

$\left.\left(\frac{\bar{X}}{\bar{X}-\rho_{i}}\right) \frac{1}{\bar{Y} \bar{X}} \sum_{i}^{k}\left(\frac{1}{n_{i}}-\frac{1}{N_{i}}\right) p_{i}^{2} \rho_{i} S_{X_{i}} S_{Y_{i}}+\left(\frac{\bar{X}}{\bar{X}-\rho_{i}}\right) \frac{1}{\bar{Y} \bar{X}} \sum_{i}^{k}\left(\frac{1}{n_{i}^{\prime}}-\frac{1}{N_{i}}\right) p_{i}^{2} \rho_{i} S_{X_{i}} S_{Y_{i}}\right]$

\section{The Mean Square Error (MSE) of $T_{a e 1}$}

The mean squared error of $T_{a e 1}$ is defined as

$\operatorname{MSE}\left(T_{a e 1}\right)=\mathrm{E}\left(T_{a e 1}-\bar{Y}\right)^{2}$

$=\mathrm{E}\left(\bar{Y}\left[-b e_{1}+b^{2} e_{1}^{2}+b e_{2}-b^{2} e_{1} e_{2}+e_{0}-b e_{0} e_{1}+b e_{0} e_{2}\right]\right)^{2}$

$=\mathrm{E}\left(\bar{Y}\left[-b e_{1}+b e_{2}+e_{0}\right] * \bar{Y}\left[-b e_{1}+b e_{2}+e_{0}\right]\right)$ since the $e_{i} \mathrm{~s}$ are neglected having power more than two.

$=\mathrm{E}\left[\bar{Y}^{2}\left(b^{2} e_{1}^{2}-2 b^{2} e_{1} e_{2}-2 b e_{0} e_{1}+b^{2} e_{2}^{2}+2 b e_{0} e_{2}+e_{0}^{2}\right)\right]$

$=\bar{Y}^{2}\left[b^{2} E\left(e_{1}^{2}\right)-b^{2} E\left(e_{1} e_{2}\right)-2 b E\left(e_{0} e_{1}\right)+2 b E\left(e_{0} e_{2}\right)+E\left(e_{0}^{2}\right)\right]$ since $\mathrm{E}\left(e_{1} e_{2}\right)=\mathrm{E}\left(e_{2}^{2}\right)$

By substituting for the expectations from section (4.1.1.)

$\operatorname{MSE}\left(T_{a e 1}\right)=\bar{Y}^{2}\left[b^{2} E\left(e_{1}^{2}\right)-b^{2} E\left(e_{1} e_{2}\right)-2 b E\left(e_{0} e_{1}\right)+2 b E\left(e_{0} e_{2}\right)+E\left(e_{0}^{2}\right)\right]$

$=\bar{Y}^{2}\left[b^{2} \frac{1}{\bar{X}^{2}} \sum_{i}^{k}\left[\left(\frac{1}{n_{i}}-\frac{1}{N_{i}}\right) p_{i}^{2} S_{X_{i}}^{2}\right]-b^{2} \frac{1}{\bar{X}^{2}} \sum_{i}^{k}\left[\left(\frac{1}{n_{i}^{\prime}}-\frac{1}{N_{i}}\right) p_{i}^{2} S_{X_{i}}^{2}\right]-2 b \frac{1}{\bar{Y} \bar{X}} \sum_{i}^{k}\left(\frac{1}{n_{i}}-\right.\right.$

$\left.\frac{1}{N_{i}}\right) p_{i}^{2} \rho_{i} S_{X_{i}} S_{Y_{i}}+2 b \frac{1}{\bar{Y} \bar{X}} \sum_{i}^{k}\left(\frac{1}{n_{i}^{\prime}}-\frac{1}{N_{i}}\right) p_{i}^{2} \rho_{i} S_{X_{i}} S_{Y_{i}}+\frac{1}{\bar{Y}^{2}} \sum_{i}^{k}\left[\left(\frac{1}{n_{i}}-\frac{1}{N_{i}}\right) p_{i}^{2} S_{Y_{i}}^{2}+\right.$

$\left.\left.\frac{\left(L_{i}-1\right)}{n_{i}} p_{i}^{2} W_{i 2} S_{Y_{i 2}}^{2}\right]\right]$

$\operatorname{MSE}\left(T_{a e 1}\right)=\left[\left(\frac{\bar{X}}{\bar{X}-\rho_{i}}\right)^{2} R^{2} \sum_{i}^{k}\left[\left(\frac{1}{n_{i}}-\frac{1}{N_{i}}\right) p_{i}^{2} S_{X_{i}}^{2}\right]-\left(\frac{\bar{X}}{\bar{X}-\rho_{i}}\right)^{2} R^{2} \sum_{i}^{k}\left[\left(\frac{1}{n_{i}^{\prime}}-\frac{1}{N_{i}}\right) p_{i}^{2} S_{X_{i}}^{2}\right]-\right.$

$2\left(\frac{\bar{X}}{\bar{X}-\rho_{i}}\right) R \sum_{i}^{k}\left(\frac{1}{n_{i}}-\frac{1}{N_{i}}\right) p_{i}^{2} \rho_{i} S_{X_{i}} S_{Y_{i}}+2\left(\frac{\bar{X}}{\bar{X}-\rho_{i}}\right) R \sum_{i}^{k}\left(\frac{1}{n_{i}^{\prime}}-\frac{1}{N_{i}}\right) p_{i}^{2} \rho_{i} S_{X_{i}} S_{Y_{i}}+\sum_{i}^{k}\left[\left(\frac{1}{n_{i}}-\right.\right.$

$\left.\left.\left.\frac{1}{N_{i}}\right) p_{i}^{2} S_{Y_{i}}^{2}+\frac{\left(L_{i}-1\right)}{n_{i}} p_{i}^{2} W_{i 2} S_{Y_{i 2}}^{2}\right]\right]$ where $\mathrm{R}=\frac{\bar{Y}}{\bar{X}}$

\section{The Optimum Values Of $n_{i}, n_{i}^{\prime}$ And $L_{i}$ for $T_{a e 1}$}

The cost function analysis adopted by Chaudhary and Kumar (2015) is used in the cost function analysis of this estimator.

Let $c_{i}^{\prime}$ be the cost per unit associated with the first phase sample of size $n_{i}^{\prime}$ and $c_{i 0}$ be the unit cost of first attempt on study variable with second phase sample of size $n_{i}$. Let $c_{i 1}$ and $c_{i 2}$ be respectively the cost per unit of enumerating the $n_{i 1}$ respondent units and $h_{i 2}$ nonrespondent units. Then the total cost for the $i^{\text {th }}$ stratum shall be given as

$$
C_{i}=c_{i}^{\prime} n_{i}^{\prime}+c_{i 0} n_{i}+c_{i 1} n_{i 1}+c_{i 2} h_{i 2} \forall \mathrm{i}=1,2, \ldots, \mathrm{k} .
$$


the expected cost per stratum is given as

$\mathrm{E}\left(C_{i}\right)=c_{i}^{\prime} n_{i}^{\prime}+n_{i}\left(c_{i 0}+c_{i 1} W_{i 1}+c_{i 2} \frac{W_{i 2}}{L_{i}}\right)$.

Thus the total cost over all the strata is represented as

$C_{0}=\sum_{i}^{k} \mathrm{E}\left(C_{i}\right)$

$=\sum_{i}^{k}\left[c_{i}^{\prime} n_{i}^{\prime}+n_{i}\left(c_{i 0}+c_{i 1} W_{i 1}+c_{i 2} \frac{W_{i 2}}{L_{i}}\right)\right]$

Given a cost function as defined as (6), the optimization function is

$\phi=\operatorname{MSE}\left(T_{a e 1}\right)+\lambda C_{0}$

where $\lambda$ is Lagrange's multiplier

$=\left[b^{2} R^{2} \sum_{i}^{k}\left[\left(\frac{1}{n_{i}}-\frac{1}{N_{i}}\right) p_{i}^{2} S_{X_{i}}^{2}\right]-b^{2} R^{2} \sum_{i}^{k}\left[\left(\frac{1}{n_{i}^{\prime}}-\frac{1}{N_{i}}\right) p_{i}^{2} S_{X_{i}}^{2}\right]-2 b R \sum_{i}^{k}\left(\frac{1}{n_{i}}-\right.\right.$

$\left.\frac{1}{N_{i}}\right) p_{i}^{2} \rho_{i} S_{X_{i}} S_{Y_{i}}+2 b R \sum_{i}^{k}\left(\frac{1}{n_{i}^{\prime}}-\frac{1}{N_{i}}\right) p_{i}^{2} \rho_{i} S_{X_{i}} S_{Y_{i}}+\sum_{i}^{k}\left[\left(\frac{1}{n_{i}}-\frac{1}{N_{i}}\right) p_{i}^{2} S_{Y_{i}}^{2}+\right.$

$\left.\left.\frac{\left(L_{i}-1\right)}{n_{i}} p_{i}^{2} W_{i 2} S_{Y_{i 2}}^{2}\right]\right]+\lambda \sum_{i}^{k}\left[c_{i}^{\prime} n_{i}^{\prime}+n_{i}\left(c_{i 0}+c_{i 1} W_{i 1}+c_{i 2} \frac{W_{i 2}}{L_{i}}\right)\right]$

In order to get the optimum values of $n_{i}, n_{i}^{\prime}$ and $L_{i}$, we differentiate $\phi$ with respect to $n_{i}, n_{i}^{\prime}$ and $L_{i}$ respectively and equate the derivatives to zero. Thus, we have for stratum i

$$
\begin{aligned}
\frac{\partial \phi}{\partial n_{i}} & =\frac{-P_{i}^{2}}{n_{i}^{2}}\left[S_{Y_{i}}^{2}+b^{2} R^{2} S_{X_{i}}^{2}-2 b R \rho_{i} S_{Y_{i}} S_{X_{i}}\right]-\frac{\left(L_{i}-1\right) W_{i 2} S_{Y_{i 2}}^{2} P_{i}^{2}}{n_{i}^{2}}+\lambda\left(c_{i 0}+c_{i 1} W_{i 1}+c_{i 2} \frac{W_{i 2}}{L_{i}}\right)=0 \\
\frac{\partial \phi}{\partial n_{i}^{\prime}} & =\frac{P_{i}^{2}}{n_{i}^{\prime 2}}\left[b^{2} R^{2} S_{X_{i}}^{2}-2 b R \rho_{i} S_{Y_{i}} S_{X_{i}}\right]+\lambda c_{i}^{\prime}=0 \\
\frac{\partial \phi}{\partial L_{i}} & =\frac{P_{i}^{2}}{n_{i}}\left[W_{i 2} S_{Y_{i 2}}^{2}\right]-\lambda n_{i} c_{i 2} \frac{W_{i 2}}{L_{i}^{2}}=0
\end{aligned}
$$

From (8)

$$
\begin{aligned}
& \lambda\left(c_{i 0}+c_{i 1} W_{i 1}+c_{i 2} \frac{W_{i 2}}{L_{i}}\right)=\frac{P_{i}^{2}}{n_{i}^{2}}\left[S_{Y_{i}}^{2}+b^{2} R^{2} S_{X_{i}}^{2}-2 b R \rho_{i} S_{Y_{i}} S_{X_{i}}+\left(L_{i}-1\right) W_{i 2} S_{Y_{i 2}}^{2}\right] \\
& n_{i}=\frac{P_{i} \sqrt{S_{Y_{i}}^{2}+b^{2} R^{2} S_{X_{i}}^{2}-2 b R \rho_{i} S_{Y_{i}} S_{X_{i}}+\left(L_{i}-1\right) W_{i 2} S_{Y_{i 2}}^{2}}}{\sqrt{\lambda\left(c_{i 0}+c_{i 1} W_{i 1}+c_{i 2} \frac{W_{i 2}}{L_{i}}\right)}}
\end{aligned}
$$

From (9)

$$
\begin{aligned}
& \lambda c_{i}^{\prime}=\frac{P_{i}^{2}}{n_{i}^{\prime 2}}\left[2 b R \rho_{i} S_{Y_{i}} S_{X_{i}}-b^{2} R^{2} S_{X_{i}}^{2}\right] \\
& n_{i}^{\prime}=\frac{P_{i} \sqrt{Q}}{\sqrt{\lambda c_{i}^{\prime}}}
\end{aligned}
$$


where $\mathrm{Q}=2 b R \rho_{i} S_{Y_{i}} S_{X_{i}}-b^{2} R^{2} S_{X_{i}}^{2}$

From (10)

$\lambda=\frac{L_{i}^{2} P_{i}^{2} S_{Y_{i 2}}^{2}}{n_{i}^{2} c_{i 2}}$ so that $\sqrt{\lambda}=\frac{L_{i} P_{i} S_{Y_{i 2}}}{n_{i} \sqrt{C_{i 2}}}$

putting the value of $\sqrt{\lambda}$ in (13) into (11), we get

$n_{i}=\frac{P_{i} \sqrt{S_{Y_{i}}^{2}+b^{2} R^{2} S_{X_{i}}^{2}-2 b R \rho_{i} S_{Y_{i}} S_{X_{i}}+\left(L_{i}-1\right) W_{i 2} S_{Y_{i 2}}^{2}}}{\frac{L_{i} P_{i} S_{Y_{i 2}}}{n_{i \sqrt{ } \sqrt{C_{i 2}}}} \sqrt{\left(c_{i 0}+c_{i 1} W_{i 1}+c_{i 2} \frac{W_{i 2}}{L_{i}}\right)}}$

$L_{i} S_{Y_{i 2}} \sqrt{\left(c_{i 0}+c_{i 1} W_{i 1}+c_{i 2} \frac{W_{i 2}}{L_{i}}\right)}=\sqrt{\left(S_{Y_{i}}^{2}+b^{2} R^{2} S_{X_{i}}^{2}-2 b R \rho_{i} S_{Y_{i}} S_{X_{i}}+\left(L_{i}-1\right) W_{i 2} S_{Y_{i 2}}^{2}\right) c_{i 2}}$

Squaring both sides, we have

$L_{i}^{2} S_{Y_{i 2}}^{2}\left(c_{i 0}+c_{i 1} W_{i 1}+c_{i 2} \frac{W_{i 2}}{L_{i}}\right)=\left(S_{Y_{i}}^{2}+b^{2} R^{2} S_{X_{i}}^{2}-2 b R \rho_{i} S_{Y_{i}} S_{X_{i}}+\left(L_{i}-1\right) W_{i 2} S_{Y_{i 2}}^{2}\right) c_{i 2}$

$L_{i}^{2} S_{Y_{i 2}}^{2} c_{i 0}+L_{i}^{2} S_{Y_{i 2}}^{2} c_{i 1} W_{i 1}+S_{Y_{i 2}}^{2} c_{i 2} L_{i} W_{i 2}-S_{Y_{i 2}}^{2} c_{i 2} L_{i} W_{i 2}=\left(S_{Y_{i}}^{2}+b^{2} R^{2} S_{X_{i}}^{2}-2 b R \rho_{i} S_{Y_{i}} S_{X_{i}}-\right.$ $\left.W_{i 2} S_{Y_{i 2}}^{2}\right) c_{i 2}$

$L_{i}^{2} S_{Y_{i 2}}^{2}\left(c_{i 0}+c_{i 1} W_{i 1}\right)=\left(S_{Y_{i}}^{2}+b^{2} R^{2} S_{X_{i}}^{2}-2 b R \rho_{i} S_{Y_{i}} S_{X_{i}}-W_{i 2} S_{Y_{i 2}}^{2}\right) c_{i 2}$

$L_{i(o p t)}=\frac{\sqrt{c_{i 2}} B_{i}}{S_{Y_{i 2} A_{i}}}$

where $A_{i}=\sqrt{c_{i 0}+c_{i 1} W_{i 1}}$;

$B_{i}=\sqrt{S_{Y_{i}}^{2}+b^{2} R^{2} S_{X_{i}}^{2}-2 b R \rho_{i} S_{Y_{i}} S_{X_{i}}-W_{i 2} S_{Y_{i 2}}^{2}}$

On substituting the value of $L_{i(o p t)}$ from (14) into (11), we can express $n_{i}$ as

$n_{i}=\frac{P_{i} \sqrt{S_{Y_{i}}^{2}+b^{2} R^{2} S_{X_{i}}^{2}-2 b R \rho_{i} S_{Y_{i}} S_{X_{i}}+\left(\frac{\sqrt{c_{i 2}} B_{i}}{S_{Y_{i 2}} A_{i}}-1\right) W_{i 2} S_{Y_{i 2}}^{2}}}{\sqrt{\lambda\left(c_{i 0}+c_{i 1} W_{i 1}+c_{i 2} \frac{W_{i 2}}{\left.\frac{\sqrt{c_{i 2} B_{i}}}{S_{Y_{i 2}} A_{i}}\right)}\right.}}$

$n_{i}=\frac{P_{i} \sqrt{S_{Y_{i}}^{2}+b^{2} R^{2} S_{X_{i}}^{2}-2 b R \rho_{i} S_{Y_{i}} S_{X_{i}}-W_{i 2} S_{Y_{i 2}}^{2}+\left(\frac{\sqrt{c_{i 2}} B_{i} S_{Y_{i 2}} W_{i 2}}{A_{i}}\right)}}{\sqrt{\lambda\left(c_{i 0}+c_{i 1} W_{i 1}+\sqrt{c_{i 2}} \frac{W_{i 2} S_{Y_{i 2} A_{i}}}{B_{i}}\right)}}$ 


$$
n_{i}=\frac{P_{i} \sqrt{B_{i}^{2}+\frac{\sqrt{c_{i 2}} B_{i} W_{i 2} S_{Y_{i 2}}}{A_{i}}}}{\sqrt{\lambda} \sqrt{A_{i}^{2}+\frac{\sqrt{c_{i 2}} A_{i} W_{i 2} S_{Y_{i 2}}}{B_{i}}}}
$$

To obtain the value of $\sqrt{\lambda}$ in terms of total $\operatorname{cost} C_{0}$, we put the value of $n_{i}, n_{i}^{\prime}$ and $L_{i(\text { opt }}$ from (15), (12) and (14) into (6), thus we get

$$
\begin{aligned}
& C_{0}=\sum_{i}^{k}\left[c_{i}^{\prime} n_{i}^{\prime}+n_{i}\left(c_{i 0}+c_{i 1} W_{i 1}+c_{i 2} \frac{W_{i 2}}{L_{i}}\right)\right] \\
& =\sum_{i}^{k}\left[c_{i}^{\prime} \frac{P_{i} \sqrt{Q}}{\sqrt{\lambda c_{i}^{\prime}}}+\frac{P_{i} \sqrt{B_{i}^{2}+\frac{\sqrt{c_{i 2}} B_{i} W_{i 2} S_{Y_{i 2}}}{A_{i}}}}{\sqrt{\lambda} \sqrt{A_{i}^{2}+\frac{\sqrt{c_{i 2}} A_{i} W_{i 2} S_{Y_{i 2}}}{B_{i}}}}\left(c_{i 0}+c_{i 1} W_{i 1}+c_{i 2} \frac{W_{i 2}}{\frac{\sqrt{c_{i 2} B_{i}}}{S_{Y_{i 2} A_{i}}}}\right)\right] \\
& =\sum_{i}^{k}\left[\sqrt{c_{i}^{\prime}} \frac{P_{i} \sqrt{Q}}{\sqrt{\lambda}}+\frac{P_{i} \sqrt{B_{i}^{2}+\frac{\sqrt{c_{i 2}} B_{i} W_{i 2} S_{Y_{i 2}}}{A_{i}}}}{\sqrt{\lambda} \sqrt{A_{i}^{2}+\frac{\sqrt{c_{i 2} A_{i} W_{i 2} S_{Y_{i 2}}}}{B_{i}}}}\left(c_{i 0}+c_{i 1} W_{i 1}+\frac{\sqrt{c_{i 2}} A_{i} W_{i 2} S_{Y_{i 2}}}{B_{i}}\right)\right] \\
& =\sum_{i}^{k}\left[\sqrt{c_{i}^{\prime}} \frac{P_{i} \sqrt{Q}}{\sqrt{\lambda}}+\frac{P_{i} \sqrt{B_{i}^{2}+\frac{\sqrt{c_{i 2}} B_{i} W_{i 2} S_{Y_{i 2}}}{A_{i}}}}{\sqrt{\lambda} \sqrt{A_{i}^{2}+\frac{\sqrt{c_{i 2}} A_{i} W_{i 2} S_{Y_{i 2}}}{B_{i}}}}\left(A_{i}^{2}+\frac{\sqrt{c_{i 2}} A_{i} W_{i 2} S_{Y_{i 2}}}{B_{i}}\right)\right] \\
& \sqrt{\lambda}=\frac{1}{C_{0}} \sum_{i}^{k}\left[\sqrt{c_{i}^{\prime}} P_{i} \sqrt{Q}+P_{i} \sqrt{B_{i}^{2}+\frac{\sqrt{c_{i 2}} B_{i} W_{i 2} S_{Y_{i 2}}}{A_{i}}} \sqrt{A_{i}^{2}+\frac{\sqrt{c_{i 2}} A_{i} W_{i 2} S_{Y_{i 2}}}{B_{i}}}\right] \\
& =\frac{1}{c_{0}} \sum_{i}^{k}\left[\sqrt{c_{i}^{\prime}} P_{i} \sqrt{Q}+P_{i} \sqrt{\left(B_{i}^{2}+\frac{\sqrt{c_{i 2}} B_{i} W_{i 2} S_{Y_{i 2}}}{A_{i}}\right)\left(A_{i}^{2}+\frac{\sqrt{c_{i 2}} A_{i} W_{i 2} S_{Y_{i 2}}}{B_{i}}\right)}\right] \\
& =\frac{1}{C_{0}} \sum_{i}^{k}\left[\sqrt{c_{i}^{\prime}} P_{i} \sqrt{Q}+P_{i} \sqrt{\frac{B_{i}^{3} A_{i}^{3}+2 \sqrt{c_{i 2}} W_{i 2} B_{i}^{2} A_{i}^{2} S_{Y_{i 2}}+c_{i 2} B_{i} A_{i} W_{i 2}^{2} S_{Y}^{2}}{B_{i} A_{i}}}\right] \\
& =\frac{1}{c_{0}} \sum_{i}^{k}\left[\sqrt{c_{i}^{\prime}} P_{i} \sqrt{Q}+P_{i} \sqrt{B_{i}^{2} A_{i}^{2}+2 \sqrt{c_{i 2}} B_{i} A_{i} W_{i 2} S_{Y_{i 2}}+c_{i 2} W_{i 2}^{2} S_{Y_{i 2}}^{2}}\right] \\
& =\frac{1}{c_{0}} \sum_{i}^{k}\left[\sqrt{c_{i}^{\prime}} P_{i} \sqrt{Q}+P_{i} \sqrt{\left(B_{i} A_{i}+\sqrt{c_{i 2}} W_{i 2} S_{Y_{i 2}}\right)^{2}}\right] \\
& \sqrt{\lambda}=\frac{1}{C_{0}} \sum_{i}^{k}\left[P_{i} \sqrt{c_{i}^{\prime}} \sqrt{Q}+P_{i}\left(B_{i} A_{i}+\sqrt{c_{i 2}} W_{i 2} S_{Y_{i 2}}\right)\right]
\end{aligned}
$$


Substituting the value of $\sqrt{\lambda}$ from (16) into (15) and (12), we respectively get the optimum values of $n_{i}$ and $n_{i}^{\prime}$ as

$$
\begin{aligned}
& n_{i(\text { opt })}=\frac{P_{i} C_{0} \sqrt{B_{i}^{2}+\frac{\sqrt{c_{i 2}} B_{i} W_{i 2} S_{Y_{i 2}}}{A_{i}}}}{\sqrt{A_{i}^{2}+\frac{\sqrt{c_{i 2}} A_{i} W_{i 2} S_{Y_{i 2}}}{B_{i}}} \sum_{i}^{k}\left[P_{i} \sqrt{c_{i}^{\prime}} \sqrt{Q}+P_{i}\left(B_{i} A_{i}+\sqrt{c_{i 2}} W_{i 2} S_{Y_{i 2}}\right)\right]} \\
& n_{i(\text { opt })}^{\prime}=\frac{P_{i} c_{0} \sqrt{Q}}{\sqrt{c_{i}^{\prime}} \sum_{i}^{k}\left[P_{i} \sqrt{c_{i}^{\prime}} \sqrt{Q}+P_{i}\left(B_{i} A_{i}+\sqrt{c_{i 2}} W_{i 2} S_{Y_{i 2}}\right)\right]}
\end{aligned}
$$

\section{Empirical Study}

Using the data set used by Chaudhary and Kumar 2015) shown on table 1 below

\section{Table 1}

\begin{tabular}{|l|l|l|l|l|l|l|l|l|l|}
\hline $\begin{array}{l}\text { Stratum } \\
\text { no }\end{array}$ & $N_{i}$ & $n_{i}^{\prime}$ & $n_{i}$ & \multicolumn{1}{|c|}{$\bar{Y}_{i}$} & \multicolumn{1}{|c|}{$\bar{X}_{i}$} & \multicolumn{1}{|c|}{$S_{Y_{i}}^{2}$} & $S_{X_{i}}^{2}$ & $\rho_{i}$ & $S_{Y_{i 2}}^{2}$ \\
\hline 1 & 73 & 65 & 26 & 40.85 & 39.56 & 6369.1 & 6624.44 & 0.999 & 618.88 \\
\hline 2 & 70 & 25 & 10 & 27.57 & 27.57 & 1051.07 & 1147.01 & 0.998 & 240.91 \\
\hline 3 & 97 & 48 & 19 & 25.44 & 25.44 & 2014.97 & 2205.4 & 0.999 & 265.52 \\
\hline 4 & 44 & 11 & 5 & 20.36 & 20.36 & 538.47 & 485.27 & 0.997 & 83.69 \\
\hline
\end{tabular}

Table 2. Below shows the Mean Squared Error (MSE) and Percent Relative Efficiency (PRE) of the proposed estimator with respect to $\bar{y}_{s t}^{*}$ for the different choices $W_{i 2}$ and $L_{i}$

\begin{tabular}{|c|c|c|c|c|c|c|c|}
\hline$W_{i 2}$ & $L_{i}$ & $\mathrm{~V}\left(\bar{y}_{s t}^{*}\right)$ & $\operatorname{MSE}\left(T_{a e 1}\right)$ & $\operatorname{MSE}\left(T_{c}^{\prime}\right)$ & $\operatorname{MSE}\left(T_{a e}\right)$ & $\operatorname{PRE}\left(T_{a e}\right)$ & $\operatorname{PRE}\left(T_{a e 1}\right)$ \\
\hline \multirow[t]{3}{*}{0.1} & 2 & 34.42 & 4.38 & 6.28 & 4.66 & 738.6 & 785.8 \\
\hline & 2.5 & 34.67 & 4.64 & 6.54 & 4.92 & 704.7 & 747.2 \\
\hline & 3 & 4.92 & 4.89 & 6.79 & 5.17 & 675.4 & 714.1 \\
\hline \multirow[t]{3}{*}{0.2} & 2 & 34.92 & 4.89 & 6.79 & 5.26 & 663.9 & 714.1 \\
\hline & 2.5 & 35.43 & 5.64 & 7.3 & 5.66 & 625.97 & 628.2 \\
\hline & 3 & 35.94 & 6.12 & 7.8 & 6.18 & 581.6 & 587.3 \\
\hline \multirow[t]{3}{*}{0.3} & 2 & 35.43 & 5.40 & 7.3 & 5.66 & 625.97 & 656.1 \\
\hline & 2.5 & 36.19 & 6.43 & 8.06 & 6.44 & 561.96 & 562.8 \\
\hline & 3 & 36.95 & 7.12 & 8.82 & 7.22 & 511.8 & 518.9 \\
\hline
\end{tabular}

\section{Table 2}




\section{CONCLUSION}

The optimum values of first phase sample size $n_{i}^{\prime}$, second phase sample size $n_{i}$ and inverse sampling rate $L_{i}$ for the different strata using the proposed estimator have been determined under the cost survey. From Table 2 it is seen that the proposed estimator is more efficient than other existing estimators, hence, the proposed estimator is recommended for use in practice.

\section{REFERENCES}

Anieting, A. E. and Enang, E. I. (2020) Two-Phase Stratified Sampling Estimator for Population Mean in the Presence of Nonresponse Using One Auxiliary Variable. Math. J. Interdiscip. Sci.Vol. 8, No.2.pp.49-56

Cochran, W. G. (1977). Sampling Techniques. New York: Wiley and SonsKhare, B. B. \& Srivastava, S. (1997).Transformed ratio type estimators for the population mean in the presence of Nonresponse. Comminication in Statistics - Theory and Methods, 26 (7), 1779-1791.

M. H. Hansen, W. N. Hurwitz (1946). The problem of non-response in sample surveys. Journal of The American Statistical Association, , no. 41, pp. 517-529.

M. K. Chaudhary and A. Kumar(2015) Use of double sample scheme in estimating the mean of Stratified population Under nonresponse. STATISTICA, anno, Lxxv,n.4

M. K. Chaudhary, R. Singh, R. K. Shukla, M. Kumar (2009). A family of estimators for estimating population mean in stratified sampling under non- response. Pakistan Journal of Statistics and Operation Research, 5, no. 1, pp. 47-54.

M. K. Chaudhary, V. K. Singh (2013). Estimating the population mean in two- stage sampling with equal size clusters under non-response using auxiliary characteristic. Mathematical Journal of Interdisciplinary Sciences, 2, no. 1, pp. 43-55.

M. K. Chaudhary, V. K. Singh, R. K. Shukla (2012). Combined-type fam- ily of estimators of population mean in stratified random sampling under non-response. Journal of Reliability and Statistical Studies (JRSS), 5, no. 2, pp. 133-142.

M. Khoshnevisan, R. Singh, P. Chauhan, N. Sawanand, F. Smarandache (2007). A general family of estimators for estimating population mean using known value of some population parameter(s). Far East Journal of Theoretical Statistics, 22, pp. 181-191.

Singer, E (2006) Introduction: Nonresponse Bias in Household Survey. The Public Opinion Quarterly. 70(5) 637-645. 